\title{
Image Enhancement Using Homomorphic Filtering and Adaptive Median Filtering for Balinese Papyrus (Lontar)
}

\author{
Ida Bagus Ketut Surya Arnawa \\ Stmik Stikom Bali \\ Bali, Indonesia
}

\begin{abstract}
Balinese papyrus (Lontar) is one of the most popular media to write for more than a hundred years in Indonesia. Balinese papyrus are used to document things that are considered important in the past. Most of the balinese papyrus suffered damage caused by weathering, edible fungus and insects making it is difficult to read. One of the efforts made to preserve the existence of balinese papyrus is to perform digitization of it. The problems most often encountered in the process of digitizing the image of the balinese papyrus is less good results as there is noise caused by its conditions that have been damaged and the uneven distribution illumination in this part of the image. In this study the authors propose to combine homomorphic filtering with adaptive median filtering to perform image enhancement. Surve results obtained show the percentage of the average respondents stated that the image enhancement results are good is $83.4 \%$, the percentage of the average respondents stated that the image enhancement results are very good is $4 \%$ and the percentage of the average respondents stated that the image enhancement results are enough is $12,6 \%$.
\end{abstract}

Keywords-Image Enhancement; Balinese Papyrus; Homomorphic Filtering; Adaptive Median Filtering; Otsu Binarization

\section{INTRODUCTION}

Balinese papyrus (Lontar) is one of the most popular media to write for more than a hundred years in Indonesia. Balinese papyrus used to document things that are considered important in the past. The tools used for writing on balinese papyrus is pengutik / pengrupak. Pengutik / Pengrupak made of steel or iron is a long rectangular, measuring $2 \times 15 \mathrm{~cm}$ with a thickness between $1.5 \mathrm{~mm}$ to $2 \mathrm{~mm}$. The content in balinese papyrus containing spell, as religious teachings, history, stories, songs, knowledge of astronomy and astrology (Wariga), philosophy of life and many other useful knowledge for us as a guide in conducting life [1].

Most of the balinese papyrus in Indonesia is currently experiencing damage caused by weathering, edible fungus and insects so it is difficult to read [1]. To preserve the balinese papyrus various attempts have been made either by the government or other institutions. One of the efforts made to preserve the existence of balinese papyrus is to perform scanning or imaging utilizing digital photos to make balinese papyrus as a digital image. In some cases the process of digitizing the balinese papyrus produces digital images that are less than good so it is necessary to perform image enhancement.

A fairly complete review is given by S.R.Yahya et al. on the method of image enhancement of an old manuscript with the background conditions that have been damaged [2]. In outline there are three types of image enhancement method, they are (a) image enhancement and thresholding binarization method, (b) image enhancement with hybridization methods between binarization / thresholding with other methods, and (c) image enhancement without thresholding method. Results of a review from [2] explains that the second method can improve image enhancement. Image Enhancement of the palm leaf manuscript using normalization techniques proposed by Z. Shi et al. [3]. Z. Shi et al. propose a set of transformation methods for processing image enhancement on a palm leaf manuscript. The first model to perform background color approximation with linear and non-linear models. Normalization techniques conducted adaptively in certain local areas. Z. Shi et al. proposed technique generates pretty good image enhancement, but there are still a lot of noise left on the final results.

The problems most often encountered in the process of digitizing the image of the balinese papyrus is there are less good results as there is noise caused by the balinese papyrus conditions that have been damaged and the uneven distribution illumination in this part of the image [4]. Deployment of uneven illumination caused by the balinese papyrus conditions that have been warped or damaged and are not allowed to use the scanner in the process of digitization. To do digitizing on the curved or broken papyrus is to use photographic equipment so that there is a distance between the papyrus with photographic equipment that result in uneven illumination. In this study the authors propose to combine homomorphic filtering with adaptive median filtering to perform image enhancement. Homomorphic filtering is used to normalize the uneven background and adaptive median filtering is used to eliminate noise found on balinese papyrus. Image that has been processed with a combination of homomorphic filtering with adaptive median filtering then binarized with global binarization using otsu binarization method without the image is divided into local images. 


\section{LITERATURE REVIEW}

\section{A. Papyrus (Lontar)}

Papyrus is one heritage spiritual wealth of the archipelago which has a very important meaning and strategic [5]. Bali is one of the places in the archipelago known to find papyrus, but some were found in Sulawesi, Java and Lombok. Before the paper is founded, papyrus is one of media that is used for writing and documenting the various things that are considered important in the past [5]. Besides papyrus as the media for writing there was also found other media such as Java used nipa leaf (similar papyrus), perkamen (skin of goat) and dluwang (skin of kind of wood). The tools used for writing on balinese papyrus is pengutik / pengrupak. It is made of steel or iron is a long rectangular, measuring $2 \times 15 \mathrm{~cm}$ with a thickness between $1.5 \mathrm{~mm}$ to $2 \mathrm{~mm}$. In the papyrus contained spells, as religious teachings, history, stories, songs, knowledge of astronomy and astrology (Wariga), philosophy of life and many other knowledge [1]. Fig. 1 is an example of papyrus.

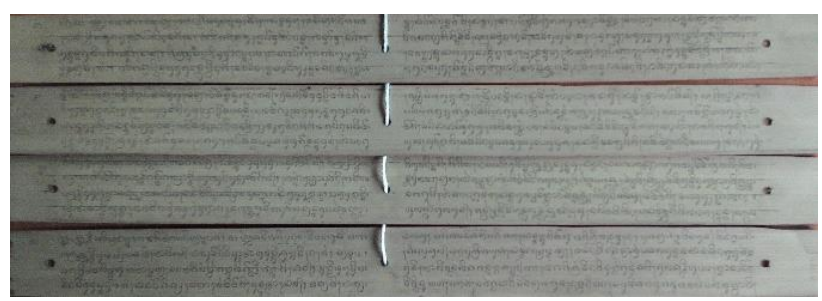

Fig. 1. Balinese Papyrus Warigasari Kyastapaka

\section{B. Homomorphic Filtering}

In image processing, homomorphic filtering is one method that can be used to compensate for the effects from uneven illumination on the image and enhance the appearance of simultaneous image compression varying intensity and contrast enhancement [6], [7]. According to this model, an image has the following equation:

$$
f(x, y)=i(x, y) r(x, y)
$$

where $f(x, y)$ is an image that is the result of multiplication (product) from $i(x, y)$ which is a component of illumination with $r(x, y)$ which is a component reflectance. To separate two independent components and facilitate their separate processing, logarithm transform on (1) has been taken, Thus

$$
\begin{aligned}
& z(x, y)=\ln f(x, y) \\
= & \ln i(x, y)+\ln r(x, y),
\end{aligned}
$$

then, the Fourier transform of (3) is calculated:

$$
\begin{aligned}
& \mathfrak{I}\{z(x, y)\}=\mathfrak{I}\{\ln f(x, y)\} \\
= & \mathfrak{I}\{\ln i(x, y)\}+\mathfrak{I}\{\ln r(x, y)\}
\end{aligned}
$$

or

$$
Z(u, v)=F_{i}(u, v)+F_{r}(u, v)
$$

where $F_{i}(u, v)$ and $F_{r}(u, v)$ is the Fourier transform from $\ln i(x, y)$ and $\ln r(x, y)$. After being moved in the frequency domain, then the image is processed by using appropriate filters so that the initial goal can be achieved is to weaken the low frequency and high frequency amplify resulting in image enhancement and image sharpening by the formula:

$$
\begin{gathered}
S(u, v)=\mathrm{H}(u, v) Z(u, v) \\
=H(u, v) F_{i}(u, v)+H(u, v) F_{r}(u, v),
\end{gathered}
$$

where $S(u, v)$ is the Fourier transform from image that has been processed. So as to obtain the actual results need to be returned to the spatial domain by the formula:

$$
\begin{gathered}
S(x, y)=\mathfrak{I}^{-1}\{S(u, v)\} \\
=\mathfrak{J}^{-1}\left\{H(u, v) F_{i}(u, v)\right\}+\mathfrak{I}^{-1}\left\{H(u, v) F_{r}(u, v)\right\}
\end{gathered}
$$

by defining

$$
i^{\prime}(x, y)=\mathfrak{J}^{-1}\left\{H(u, v) F_{i}(u, v)\right\}
$$

and

$$
r^{\prime}(x, y)=\mathfrak{J}^{-1}\left\{H(u, v) F_{r}(u, v)\right\}
$$

Equation (10) can be expressed as follows:

$$
s(x, y)=i^{\prime}(x, y)+r^{\prime}(x, y) \text {, }
$$

the final step is to eliminate logarithms operations conducted at the beginning of the process by performing an exponential operation in order to obtain the desired enhanced image is denoted by $\mathrm{g}(x, y)$ is:

$$
\begin{aligned}
& \mathrm{g}(x, y)=e^{s(x, y)} \\
= & e^{i \prime(x, y)} e^{r^{\prime}(x, y)} \\
= & i_{0}(x, y) r_{0}(x, y),
\end{aligned}
$$

where $i_{0}(x, y)=e^{i^{\prime}(x, y)}$ and $r_{0}(x, y)=e^{r^{\prime}(x, y)}$ is the illumination and reflectance components from output each image. The $\mathrm{H}(u, v)$ normally used in this procedure is the Butterworth high pass filter defined as :

$$
H(u, v)=\left(\gamma_{H}-\gamma_{L}\right)\left(\frac{1}{1+\left(D_{0} / D(u, v)\right)^{2 n}}\right)+\gamma_{L}
$$

where $D_{0}$ is the cut off distance measured from the origin, $D(u, v)$ is distance from the origin of centered Fourier transform, and $\mathrm{n}$ is the order of the Butterworth filter.

\section{Adaptive Median Filtering}

The problem faced by the standard median filtering can be resolved with adaptive median filtering. Between the adaptive 
median filtering and median filtering have a fundamental difference in which adaptive median filtering window surrounding each pixel is variable [8]. In the standard median filter does not take into account variations in image characteristics from one point to another [9]. Flowchart from adaptive median filtering shown in Fig. 2.

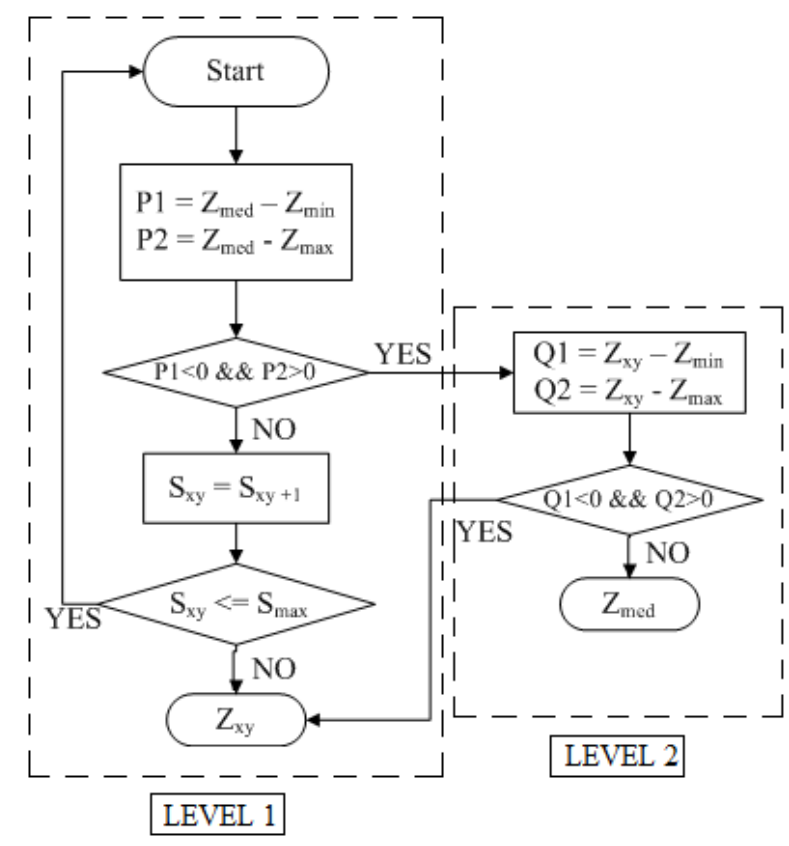

Fig. 2. Flowchart Adaptive Median Filtering

In the Fig. 2 adaptive median filtering works with 2 levels, they are level 1 and level 2. At level 1 serves to determine whether the median filter output $\mathrm{Z}_{\text {med }}$ is impulse output or not. If the impulse output is not found on the first level then it will proceed to level 2. At level 2, adaptive median filtering would increase the size of the window and repeat the process at the level of 1 to find that the median value is not impulse or the maximum window size has been reached. Adaptive median filtering can reduce computational overhead because every time the value of the output of the adaptive median filtering algorithm, $S$ xy window is moved to the next location in the image. Adaptive median filtering algorithm then performs reinitialized and applied to a pixel in a new location. Adaptive median filtering has three main objectives : repair the damaged image that is caused by salt and impulse noise, give smoothing from non-impulsive noise and reduce the disturbance caused by thinning or thickening of object excess limit [9].

\section{MeTHODOLOGY}

\section{A. Data Acquisition}

Data acquisition is the process of acquiring data from analog to digital, from balinese papyrus physically into image files by using the digital camera or scanner. Balinese papyrus were in good condition scanned using the scanner while the balinese papyrus that is in poor condition scanned using a digital camera. Results from scanning balinese papyrus stored in a computer before further processing. Balinese papyrus image used in this study can be seen in Fig. 3 (a) to (f).

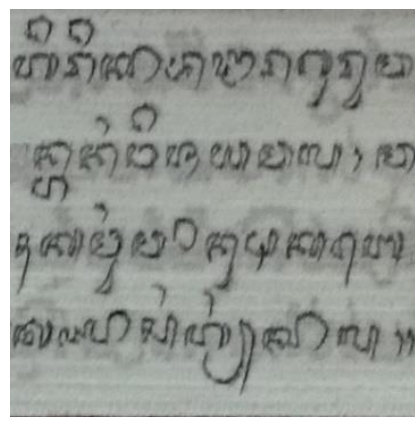

(a)

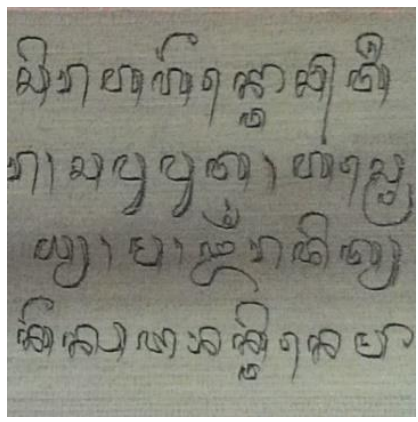

(c)

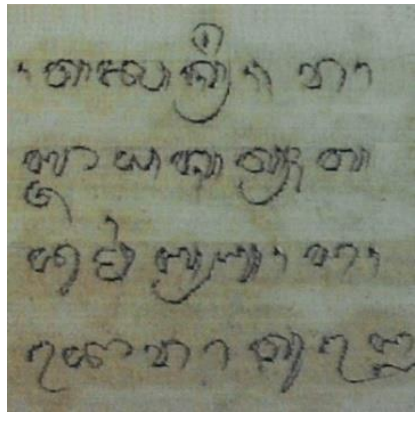

(e)

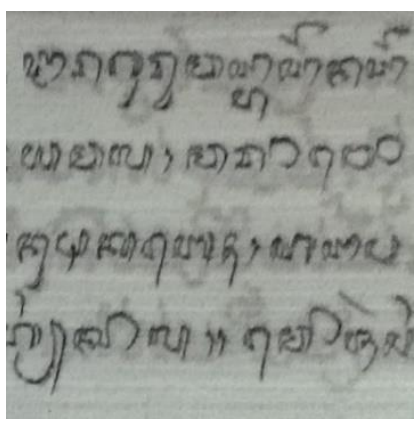

(b)

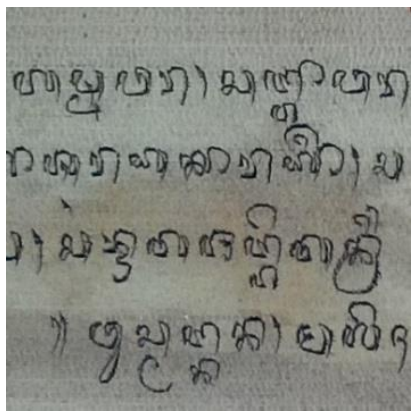

(d)

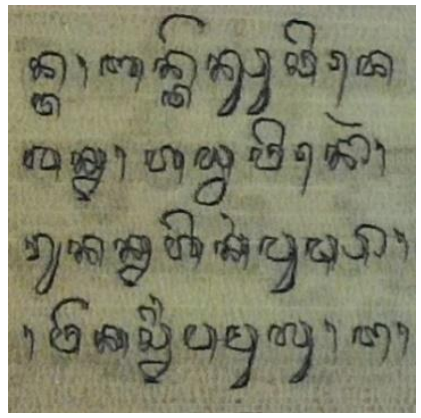

(f)
Fig. 3. Example of input images

\section{B. Algorithm}

Result from the acquisition of the data stored in the computer are divided into local images. Local images is first converted into a grayscale image. Then the result from grayscale images is filtered by using homomorphic filtering and adaptive median filtering. Filtration results of each section then are binarized with otsu binarization and the results are put back together into a complete image [10]. Next binarization results were evaluated by questionnaire to some correspondents to assess the results from image enhancement. Fig. 4 is the steps performed in this study.

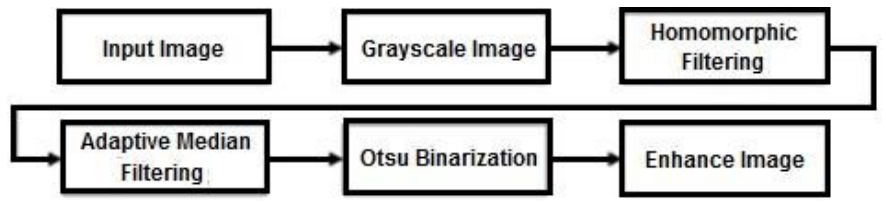

Fig. 4. Algorithm Based Process 


\section{RESUlt AND DiscUSSION}

\section{A. Result}

1) Homomorphic Filtering

Homomorphic filtering is applied in this study using a Butterworth filter that is adopted from [11]. Butterworth filter formula is shown in (17), $\gamma_{H}$ parameter value, and $\gamma_{L}$ respectively are 1.2 and 0.03 and the value of $\mathrm{n}=1$. The series of homomorphic filtering are implemented in the matlab. Results from homomorphic filtering process shown in Fig. 5. Each image in Fig. 5 (a) to (f) is the result from homomorphic filtering from the image in Fig. 3 (a) to (f).

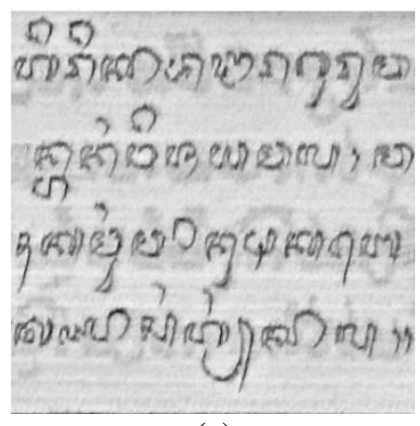

(a)

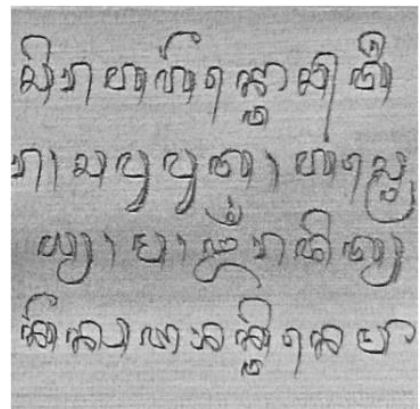

(c)

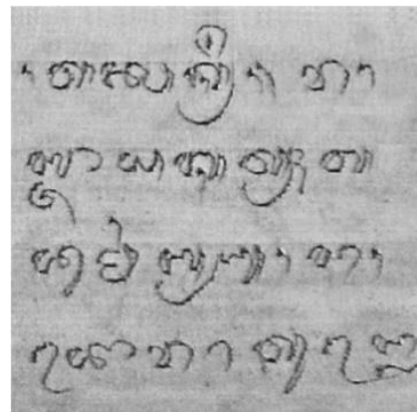

(e)

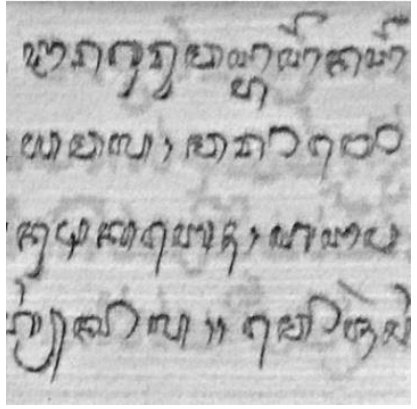

(b)

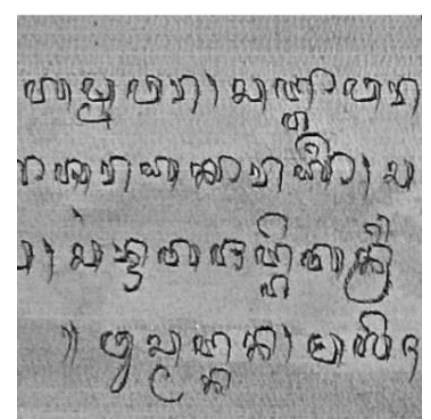

(d)

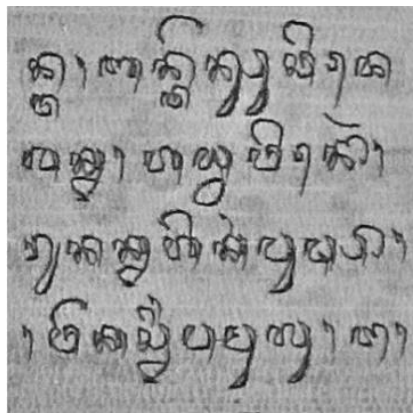

(f)
Fig. 5. Result Homomorphic Filtering

\section{2) Adaptive Median Filtering}

Adaptive median filtering is applied to the image enhancement research using ws parameter values and c respectively are 100 and 0,001 . The series of adaptive median filtering are implemented in the matlab. Results from adaptive median filtering process are shown in Fig. 6. Each image in Fig. 6 (a) to (f) is the result from adaptive median filtering from the image in Fig. 5 (a) to (f).

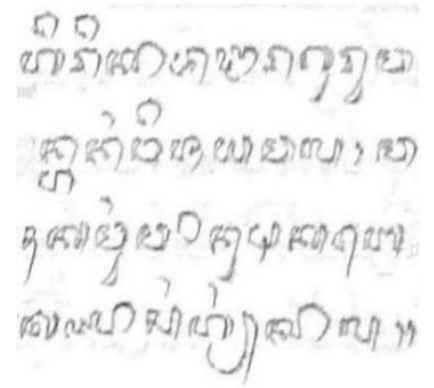

(a)

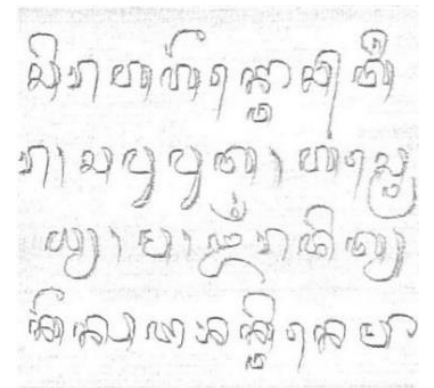

(c)

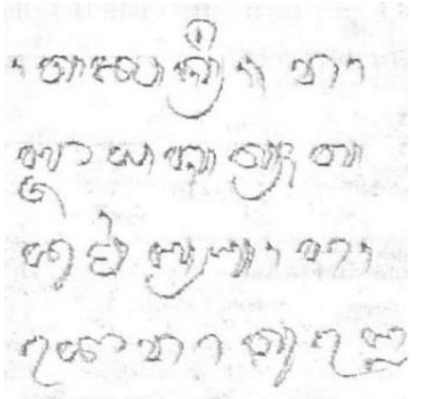

(e)

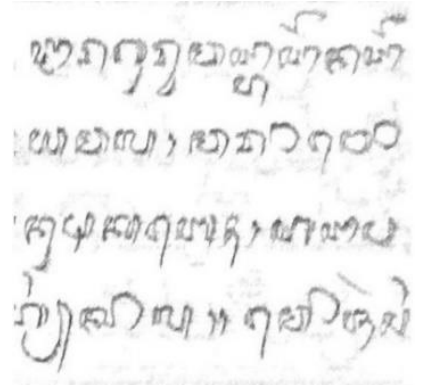

(b)

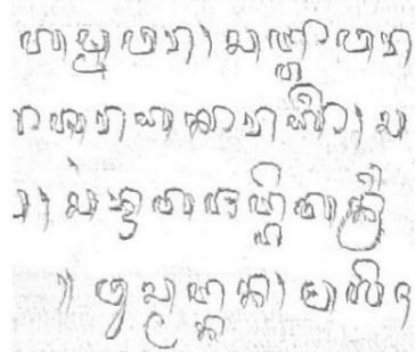

(d)

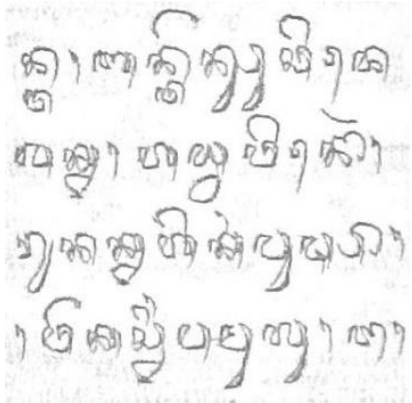

(f)
Fig. 6. Result Adaptive Median Filtering

\section{3) Otsu Binarization}

After the homomorphic filtering and adaptive median filtering, then it is followed by binarization process using otsu method [11]. Results from the process otsu binarization method shown in Fig. 7 (a) to (f).

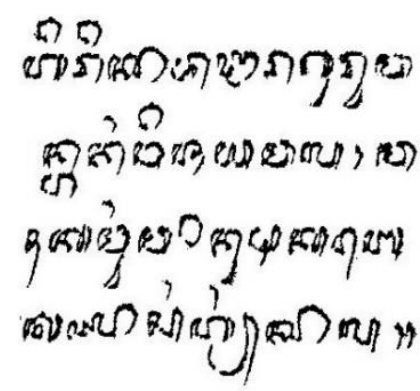

(a)

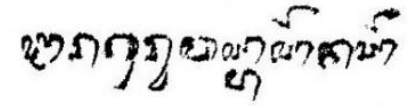

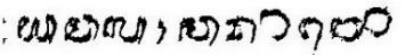

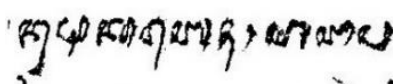

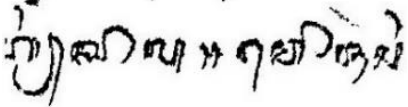

(b) 


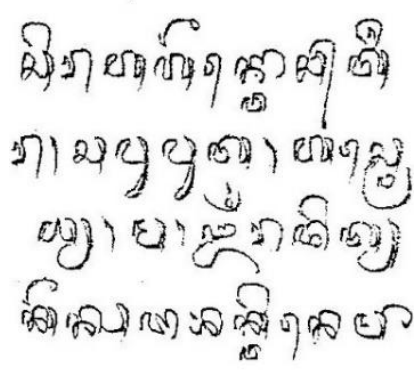

(c)

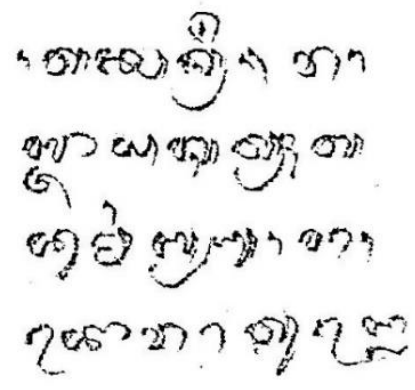

(e)

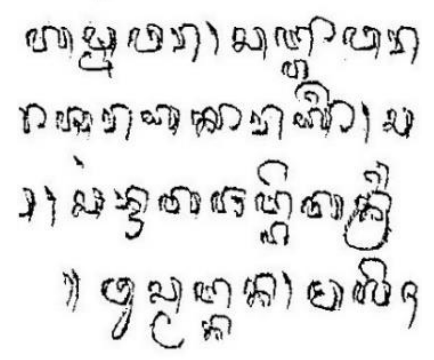

(d)

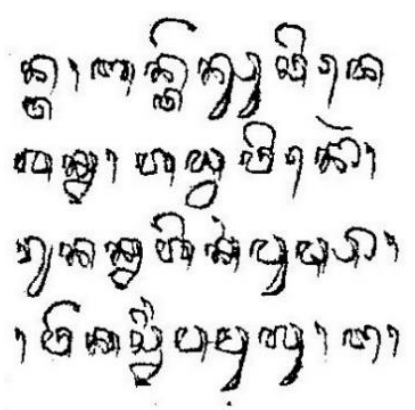

(f)
Fig. 7. Result Otsu Binarization

\section{4) Field Trial}

In the field trial, the authors tested the results from image enhancement using a questionnaire to determine the quality from the resulting image enhancement. In conducting the questionnaire, the authors involved 27 people who come from the balinese language lecturer, teacher and student of balinese language. In the process of filling in the questionnaire, authors included balinese papyrus to compare the original text which is in original balinese papyrus with enhancement of existing results in the form of questionnaires. Diagrams of score percentage of the answers given by the respondents can be described as Fig.8.

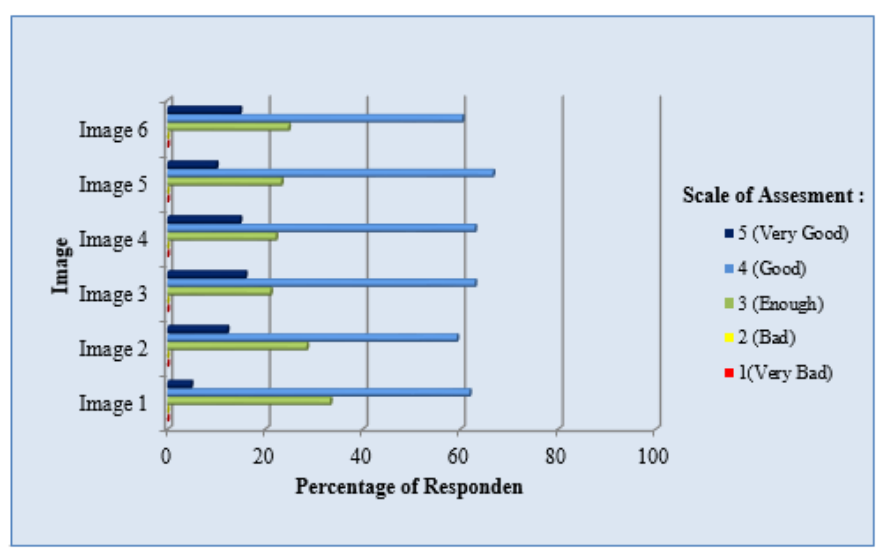

Fig. 8. Percentage Diagram of Respondents Answer Score In Field Trial

Based on the percentage of respondents answer scores diagram in field trial, it can be viewed that the test results from image enhancement is good. This is evidenced by the average percentage of stating the results from image enhancement are good is $83.4 \%$ and the average percentage of stating the results from image enhancement are excellent is $4 \%$ as well as the average percentage of stating the results from image enhancement are enough is $12.6 \%$.

\section{B. Discussion}

From the results obtained can be viewed that the homomorphic filtering and adaptive median filtering has been able to perform image enhancement at balinese papyrus. On the results of image enhancement, there are still some dotted text. Text experiencing this dot caused by the otsu binarization result from parts of the input image is categorized as part of the background and rated the white pixel so that the texts are experiencing dashed in some parts. Moreover, in the image that has a lot of noise, some parts from image are categorized includes the foreground and rated the pixel black, causing there are still some residual noise. Though the results is ideally every part of foreground converted to black and every backgound converted into white. Otsu binarization errors can be overcome by performing filtration processes using homomorphic filtering first and then the results from homomorphic filtering is filtered again using adaptive median filtering before otsu binarization process. Results from homomorphic filtering process shown in Fig. 5 and the results from adaptive median filtering shown in Fig. 6. Filtration process with homomorphic filtering and adaptive median filtering has managed to homogenize backgound and minimize the noise level so that the results from the otsu binarization process can be better in which pixels are categorized into the foreground section and the incoming pixel backgound section as shown in Fig. 7.

Testing of the results of image enhancement is done by giving questionnaires to 27 people consisting from the balinese language lecturer, teacher and student of balinese language. Results from questionnaire that has been done shows that the input image has the $5^{\text {th }}$ lowest percentage is $76.5 \%$ said good, $21.5 \%$ said enough and $2 \%$ said very good. $5^{\text {th }}$ input image is an image with a backgound which has a lot of noise and have low contrast between the foreground with the background. $5^{\text {th }}$ input image shown in Fig. 3 (e). The results are less good at the $5^{\text {th }}$ input image because some parts from foreground categorized as part of backgound and rated as white pixels, resulting in the yield on some text experiencing dashed.

\section{CONCLUSIONS}

Based on the results it can be concluded the combination of homomorphic filtering with adaptive median filtering are good for enhancing old balinese papyrus. Steps taken to perform image enhancement on balinese papyrus is to use a filtration process homomorphic filtering and adaptive median filtering and then binarization is performed by using the otsu binarization method. Results from image enhancement on balinese papyrus using homomorphic filtering and adaptive median filtering shows damaged balinese papyrus image could be improved better. Results of image enhancement was tested by conducting questionnaire to 27 people who come from the balinese language lecturer, teacher and student of balinese language and produces a high percentage for a category of good image enhancement results. In future studies a new method will be added to enhance the results obtained from the combination of homomorphic filtering with adaptive median filtering and will be tested with different case studies. 


\section{REFERENCES}

[1] M. Sudarma, "Identifying of the Cielab Space Color for the Balinese Papyrus Characters," Telkomnika Indonesian Journal of Electrical Engineering, vol. 13, pp. 321-328, February 2015.

[2] S.R. Yahya, S.N.H.S. Abdullah, K. Omar, M.S. Zakaria, and C.-Y. Liong, "Review on Image Enhancement Method of Old Manuscript with Damaged Background," in International Journal on Electrical Engineering and Informatics, vol. 2, January 2010.

[3] Z. Shi, S. Setlur, and V. Govindaraju, "Digital Enchancement of Palm Leaf Manuscript Images using Normalization Techniques," 5th International Conference On Knowledge Based Computer Systems, pp.19-22, December 2005.

[4] C.H. Chou, W.H. Lin, and F. Chang, "A Binarization Method with Learning-Built Rules for Document Images Produced by Camera" Pattern Recognition, vol.43,pp. 1518-1530, November 2009.

[5] M. Sudarma, and N.P. Sutramiani, "The Thinning Zhang-Suen Application Method in the Image of Balinese Scripts on the Papyrus," International Journal of Computer Applications, vol. 91,April 2014.

[6] R. Bock, J. Meier, L.G. Nyul, J. Hornegger, and G. Michelson, "Glaucoma Risk Index : Automated Glaucoma Detection from Color Fundus Images," Medical Image Analysis, vol.14,pp. 471-481, June 2010.
[7] R. Gonzalez, and R.E. Woods, Digital Image Processing, 3rd ed. NJ, USA: Prentice Hall, 2008.

[8] D. Dhanasekaran, A. Krishnamurthy, and Ramkumar, "High Speed Pipeline Architecture for Adaptive Median Filter," Proceedings of the International Conference on Advances in Computing, Communication and Control, pp. 591-600, 2009.

[9] S. Sarker, S. Chowdhury, S. Laha, and D. Dey, "Use of Non-Local Mean Filter to Denoise Image Corrupted by Salt and Pepper Noise," Signal \& Image Processing : An International Journal (SIPIJ), vol. 3,pp. 223-235,April 2012.

[10] N. Otsu, "A Threshold Selection Method from Gray-Level Histogram," Automatica, vol.11, pp. 23-27, 1975.

[11] H. Shahamat, and A.A. Pouyan, "Face Recognition Under Large Illumination Variations Using Homomorphic Filtering in Spatial Domain," Journal of Visual Communication and Image Representation, vol. 25, pp. 970-977, March 2014. 